\title{
REGULARARTICLE \\ BODY WEIGHT PREDICTION IN NIGERIA GOATS USING BODY MEASUREMENTS OF DIFFERENT AGE, SEX AND BREEDS
}

\author{
A. J. SHOYOMBO' ${ }^{*}$, A. A MUSA ${ }^{2}$, M. WHETO ${ }^{3}$ \\ ${ }^{1}$ Department of Animal Science, College of Agricultural Sciences, Landmark University Omu-Aran, Kwara State, Nigeria \\ ${ }^{2}$ Department of Animal Science, Faculty of Agriculture, Kogi State University, Anyigba, Kogi State, Nigeria \\ 3Department of Animal Breeding and Genetics, Federal University of Agriculture, Abeokuta, Ogun State, Nigeria
}

\begin{abstract}
Data collected from 900 goats sampled from Borno, Sokoto and Ogun States from three different breeds on the following metric characters: Body Weight (BW), Age, Horn Length (HL), Ear length (EL), Shoulder width (SW), Neck circumference (NC), body length (BL), Withers Height (WH), Heart Girth (HG), Pouch Girth (PG), Tail Length (TL) and Scrotal circumference (SC) were subjected to stepwise multiple linear regression. Results obtained showed Live weight changes with body measurements were poorly to highly predictable with $\mathrm{R}^{2}$ values ranging between $0.000-0.031$ in animals $<1 \mathrm{y}$ old, o.0o0o.241in animals 1-2 y and 0.00o-0.658 in animals $2 \mathrm{y}$ and above. It was thus concluded that the low, moderate and high predictive power obtained from this study might be due to instability of regression coefficient at different ages and points also to decreasing environmental impact on growth with increase in age.
\end{abstract}

\section{INTRODUCTION}

Predicting life weight from body measurements in livestock is taking a center stage in growth evaluation, several works have stated positive effect of chosen predictor variables in body weight determination [1-3]. Further examples of this also includes the report of Baffour-Awuah et al. [4] that body lengths, width at shoulder, heart girth were significant predictors. However, the superiority of heart girth over other linear body measurements has been reported by other workers $[1,5]$ Salako and Ngere [6] reported that heart girth and body weight can be varied depends on breed qualities as well as feed and management. Ojedapo et al. [7] reported that sexual dimorphism in body weight and other body linear measurements favored females than males in goats. However, predictors are often subject to the classification variables. This study was designed to use a comprehensive classification of goats using age, sex and breed as factors for morphometric predictors of body weight.

\section{MATERIALS AND METHODS}

Data was collected from goats in Borno, Sokoto and Ogun States. These states were selected because they are locations having close to pure breeds of the goats. Animals used for this study were sampled in the abattoir, of Borno, Sokoto and Ogun states when brought for slaughter either by the owner or by the slaughter man. It is believed that all animals find their way into the abattoir from villages and local markets, where they are kept in small numbers by local farmers; they are raised under the extensive system of management. A total of nine hundred (900) goats comprising of three hundred Sahel goats from Borno state, three hundred Red Sokoto goats from Sokoto state and three hundred West African Dwarf goats from Ogun state were used for the study. In these breeds, 300 goats were selected. These were evaluated for morphometric characteristics. The pairs of permanent incisors in the dentition of the goat were used to determine age. The following metric characters were measured on each animal: Body Weight (BW), Age, Horn Length (HL), Ear length (EL), Shoulder width (SW), Neck circumference (NC), body length (BL), Withers Height (WH), Heart Girth (HG), Pouch Girth (PG), Tail Length (TL) and Scrotal circumference (SC). Reference marks used for body measurement according to the method of and Salako and Ngere [6]. Obtained data was subjected to stepwise multiple regression, the following linear multiple regression models were applied.

$\mathrm{Y}=\mathrm{a}+\mathrm{b}_{1} \mathrm{X}_{1}+\mathrm{b}_{2} \mathrm{X}_{2}+\mathrm{b}_{3} \mathrm{X}_{3}+\ldots \ldots \mathrm{b}_{\mathrm{i}} \mathrm{X}_{\mathrm{i}}$

Where:

$\mathrm{Y}=$ the dependent variable (Live weight)

$\mathrm{a}=$ the intercept of regression curve on $\mathrm{y}$-axis and is the value of the dependent variable $y$ when all independent variables are Zero.

$\mathrm{b}_{1}=$ the partial regression coefficient associated with respective independent variable $\mathrm{X}_{1}$.

\section{Received 14 November 2017; Accepted 10 December 2017 \\ *Corresponding Author}

\section{A J Shoyombo}

Department of Animal Science, College of Agricultural Sciences, Landmark University Omu-Aran, Kwara State, Nigeria

Email: shoyombo.ayoola@lmu.edu.ng

( This article is open access and licensed under the terms of the Creative Commons Attribution License (http://creativecommons.org/licenses/by/4.o/) which permits unrestricted, use, distribution and reproduction in any medium, or format for any purpose, even commercially provided the work is properly cited. Attribution - You must give appropriate credit, provide a link to the license, and indicate if changes were made. 
$\mathrm{X}_{1}=$ the independent variables (i.e. body measurement) the regression assumes that the

Independent variable has no measurement error. And that the above errors about the regression line are equal. The regression analysis was carried out using the SASREG procedure of the SAS [8] Package.

Table 1: Regression of liveweight on body parameters ( $Y=$ live weight) in does $<1 y e a r$ of age

\begin{tabular}{|c|c|c|c|c|}
\hline Breed & Parameter & Regression equation & $\mathbf{r}^{2}$ & Significance \\
\hline \multirow[t]{9}{*}{ Red Sokoto } & HL & $Y=12.560+0.189 x$ & 0.014 & $\mathrm{Ns}$ \\
\hline & EL & $\mathrm{Y}=11.480+0.220 \mathrm{x}$ & 0.005 & Ns \\
\hline & SW & $\mathrm{Y}=12.709+0.077 \mathrm{x}$ & 0.007 & Ns \\
\hline & $\mathrm{NC}$ & $\mathrm{Y}=12.962+0.034 \mathrm{x}$ & 0.002 & Ns \\
\hline & $\mathrm{BL}$ & $Y=13.653+0.002 x$ & 0.000 & $\mathrm{Ns}$ \\
\hline & WH & $Y=12.001+0.033 x$ & 0.003 & Ns \\
\hline & HG & $Y=11.640+0.039 x$ & 0.010 & Ns \\
\hline & PG & $\mathrm{Y}=11.55+0.039 \mathrm{x}$ & 0.010 & Ns \\
\hline & TL & $\mathrm{Y}=17.612+0.321 \mathrm{x}$ & 0.027 & Ns \\
\hline \multirow[t]{9}{*}{ Sahel } & $\mathrm{HL}$ & $Y=9.567+0.553 x$ & 0.102 & $*$ \\
\hline & EL & $\mathrm{Y}=10.559+0.260 \mathrm{x}$ & 0.014 & Ns \\
\hline & SW & $Y=3.085+0.576 x$ & 0.190 & $* *$ \\
\hline & $\mathrm{NC}$ & $\mathrm{Y}=4.928+0.411 \mathrm{x}$ & 0.143 & $* *$ \\
\hline & $\mathrm{BL}$ & $\mathrm{Y}=7.949+0.159 \mathrm{x}$ & 0.081 & $*$ \\
\hline & WH & $Y=8.616+0.116 x$ & 0.085 & $*$ \\
\hline & HG & $Y=6.514+0.141 x$ & 0.015 & Ns \\
\hline & PG & $\mathrm{Y}=6.383+0.157 \mathrm{x}$ & 0.015 & Ns \\
\hline & TL & $\mathrm{Y}=11.963+0.137 \mathrm{x}$ & 0.006 & Ns \\
\hline \multirow{9}{*}{ West African Dwarf } & $\mathrm{HL}$ & $\mathrm{Y}=10.764+0.061 \mathrm{x}$ & 0.007 & Ns \\
\hline & EL & $\mathrm{Y}=11.357+0.096 \mathrm{x}$ & 0.005 & $\mathrm{Ns}$ \\
\hline & SW & $\mathrm{Y}=9.179+0.099 \mathrm{x}$ & 0.038 & $\mathrm{Ns}$ \\
\hline & $\mathrm{NC}$ & $\mathrm{Y}=10.706+0.011 \mathrm{x}$ & 0.000 & $\mathrm{Ns}$ \\
\hline & $\mathrm{BL}$ & $\mathrm{Y}=7.119+0.079 \mathrm{x}$ & 0.092 & $*$ \\
\hline & WH & $\mathrm{Y}=10.409+0.007 \mathrm{x}$ & 0.000 & Ns \\
\hline & HG & $\mathrm{Y}=9.712+0.015 \mathrm{x}$ & 0.003 & $\mathrm{Ns}$ \\
\hline & PG & $\mathrm{Y}=9.878+0.011 \mathrm{x}$ & 0.002 & $\mathrm{Ns}$ \\
\hline & TL & $\mathrm{Y}=8.338+0.204 \mathrm{x}$ & 0.031 & Ns \\
\hline
\end{tabular}

${ }^{* *} \mathrm{P}<0.01 * \mathrm{P}<0.05$ ns-not significant

Table 2: Regression of live weight on body parameters $(Y=$ liveweight $)$ in Bucks $<1$ y

\begin{tabular}{|c|c|c|c|c|}
\hline Breed & Parameter & Regression equation & $\mathbf{r}^{2}$ & Significance \\
\hline \multirow[t]{9}{*}{ Red Sokoto } & HL & $Y=11.360+0.287 x$ & 0.023 & Ns \\
\hline & EL & $Y=10.937+0.195 x$ & 0.005 & Ns \\
\hline & SW & $Y=11.130+0.124 x$ & 0.020 & Ns \\
\hline & $\mathrm{NC}$ & $Y=13.108-0.011 x$ & 0.000 & Ns \\
\hline & $\mathrm{BL}$ & $Y=9.261+0.086 x$ & 0.013 & Ns \\
\hline & WH & $Y=0.863+0.266 x$ & 0.254 & Ns \\
\hline & HG & $Y=9.356+0.060 x$ & 0.028 & Ns \\
\hline & PG & $Y=9.337+0.059 x$ & 0.027 & Ns \\
\hline & TL & $Y=10.661+0.187 x$ & 0.017 & Ns \\
\hline \multirow[t]{9}{*}{ Sahel } & HL & $Y=9.718+0.704 x$ & 0.169 & $* *$ \\
\hline & EL & $Y=10.753+0.379 x$ & 0.037 & Ns \\
\hline & SW & $Y=5.145+0.526 x$ & 0.133 & $* *$ \\
\hline & $\mathrm{NC}$ & $Y=6.381+0.367 x$ & 0.202 & $* *$ \\
\hline & $\mathrm{BL}$ & $\mathrm{Y}=8.347+0.167 \mathrm{x}$ & 0.174 & $* *$ \\
\hline & WH & $\mathrm{Y}=7.849+0.155 \mathrm{x}$ & 0.156 & $* *$ \\
\hline & HG & $Y=20.180+0.107 x$ & 0.018 & Ns \\
\hline & PG & $Y=22.225+0.144 x$ & 0.033 & Ns \\
\hline & TL & $Y=13.961+0.082 x$ & 0.003 & Ns \\
\hline \multirow[t]{9}{*}{ West African Dwarf } & HL & $Y=1.164-0.108 x$ & 0.021 & Ns \\
\hline & EL & $\mathrm{Y}=8.421+0.234 \mathrm{x}$ & 0.035 & Ns \\
\hline & SW & $Y=9.981+0.056 x$ & 0.017 & Ns \\
\hline & $\mathrm{NC}$ & $Y=10.190+0.018 x$ & 0.003 & Ns \\
\hline & $\mathrm{BL}$ & $\mathrm{Y}=8.998+0.039 \mathrm{x}$ & 0.018 & Ns \\
\hline & WH & $Y=10.085+0.011 x$ & 0.003 & Ns \\
\hline & HG & $Y=9.138+0.029 x$ & 0.011 & Ns \\
\hline & PG & $Y=8.843+0.035 x$ & 0.014 & Ns \\
\hline & $\mathrm{TL}$ & $\mathrm{Y}=12.244+0.157 \mathrm{x}$ & 0.024 & Ns \\
\hline
\end{tabular}


Table 3: Regression of liveweight on body parameters ( $Y=$ live wieght $)$ in does at 1-2 y

\begin{tabular}{|c|c|c|c|c|}
\hline Breed & Parameter & Regression equation & $\mathbf{r}^{2}$ & Significance \\
\hline \multirow[t]{9}{*}{ Red Sokoto } & HL & $Y=12.758+0.122 x$ & 0.007 & Ns \\
\hline & EL & $Y=12.068+0.125 x$ & 0.007 & Ns \\
\hline & SW & $Y=17.156-0.266 x$ & 0.030 & Ns \\
\hline & $\mathrm{NC}$ & $\mathrm{Y}=12.028+0.061 \mathrm{x}$ & 0.005 & Ns \\
\hline & $\mathrm{BL}$ & $Y=4.091+0.187 x$ & 0.241 & Ns \\
\hline & WH & $Y=-1.343+0269 x$ & 0.202 & Ns \\
\hline & HG & $Y=13.778-0.003 x$ & 0.000 & Ns \\
\hline & PG & $Y=14.994+0.210 x$ & 0.000 & Ns \\
\hline & TL & $\mathrm{Y}=12.199+0.114 \mathrm{x}$ & 0.005 & Ns \\
\hline \multirow[t]{9}{*}{ Sahel } & HL & $\mathrm{Y}=13.592+0.375 \mathrm{x}$ & 0.005 & $*$ \\
\hline & EL & $\mathrm{Y}=10.748+0.462 \mathrm{x}$ & 0.185 & $* *$ \\
\hline & SW & $Y=17.427+0.013 x$ & 0.000 & Ns \\
\hline & $\mathrm{NC}$ & $Y=17.420+0.011 x$ & 0.000 & Ns \\
\hline & $\mathrm{BL}$ & $\mathrm{Y}=8.994+0.146 \mathrm{x}$ & 0.165 & $* *$ \\
\hline & WH & $\mathrm{Y}=5.383+0.192 \mathrm{x}$ & 0.176 & $* *$ \\
\hline & HG & $\mathrm{Y}=9.154+0.132 \mathrm{x}$ & 0.072 & * \\
\hline & PG & $\mathrm{Y}=9.051+0.128 \mathrm{x}$ & 0.069 & Ns \\
\hline & $\mathrm{TL}$ & $\mathrm{Y}=13.985+0.233 \mathrm{x}$ & 0.019 & Ns \\
\hline \multirow[t]{9}{*}{ West African Dwarf } & HL & $Y=11.279+0.669 x$ & 0.170 & $* *$ \\
\hline & EL & $\mathrm{Y}=5.765+0.860 \mathrm{x}$ & 0.122 & $* *$ \\
\hline & SW & $\mathrm{Y}=13.024+0.174 \mathrm{x}$ & 0.024 & Ns \\
\hline & $\mathrm{NC}$ & $\mathrm{Y}=18.734+0.133 \mathrm{x}$ & 0.008 & $\mathrm{Ns}$ \\
\hline & $\mathrm{BL}$ & $\mathrm{Y}=5.296+0.196 \mathrm{x}$ & 0.126 & $* *$ \\
\hline & WH & $Y=13.543+0.036 x$ & 0.005 & $\mathrm{Ns}$ \\
\hline & $\mathrm{HG}$ & $\mathrm{Y}=34.793+0.318 \mathrm{x}$ & 0.286 & $* *$ \\
\hline & PG & $\mathrm{Y}=34.250-0.301 \mathrm{x}$ & 0.309 & $\mathrm{Ns}$ \\
\hline & $\mathrm{TL}$ & $\mathrm{Y}=11.015+0.405 \mathrm{x}$ & 0.048 & $\mathrm{Ns}$ \\
\hline
\end{tabular}

${ }^{* *} \mathrm{P}<0.01,{ }^{*} \mathrm{P}<0.05$, Ns-not significant

Table 4: Regression of liveweight on body parameters ( $Y=$ live wieght) in bucks at $1-2 \mathrm{y}$ of age

\begin{tabular}{|c|c|c|c|c|}
\hline Breed & Parameter & Regression equation & $\mathbf{r}^{2}$ & Significance \\
\hline \multirow[t]{9}{*}{ Red Sokoto } & HL & $Y=9.534+0.543 x$ & 0.011 & $* *$ \\
\hline & EL & $Y=16.486-0.250 x$ & 0.034 & Ns \\
\hline & SW & $Y=13.120+0.029 x$ & 0.000 & Ns \\
\hline & $\mathrm{NC}$ & $Y=10.781+0.104 x$ & 0.025 & Ns \\
\hline & BL & $\mathrm{Y}=8.977+0.163 \mathrm{x}$ & 0.074 & $*$ \\
\hline & WH & $\mathrm{Y}=0.640+0.240 \mathrm{x}$ & 0.260 & $* *$ \\
\hline & HG & $Y=16.054-0.041 x$ & 0.005 & Ns \\
\hline & PG & $\mathrm{Y}=16.44+0.045 \mathrm{x}$ & 0.06 & Ns \\
\hline & TL & $\mathrm{Y}=11.237+0.177 \mathrm{x}$ & 0.010 & Ns \\
\hline \multirow[t]{9}{*}{ Sahel } & HL & $\mathrm{Y}=16.767+0.011 \mathrm{x}$ & 0.000 & Ns \\
\hline & EL & $\mathrm{Y}=12.204+0.364 \mathrm{x}$ & 0.078 & * \\
\hline & SW & $\mathrm{Y}=13.718+0.139 \mathrm{x}$ & 0.014 & Ns \\
\hline & $\mathrm{NC}$ & $\mathrm{Y}=9.992+0.265 \mathrm{x}$ & 0.035 & Ns \\
\hline & $\mathrm{BL}$ & $\mathrm{Y}=2.517+0.267 \mathrm{x}$ & 0.164 & $* *$ \\
\hline & WH & $Y=13.667+0.053 x$ & 0.009 & * \\
\hline & HG & $\mathrm{Y}=2.261+0.244 \mathrm{X}$ & 0.152 & $* *$ \\
\hline & PG & $\mathrm{Y}=5.475+0.184 \mathrm{X}$ & 0.127 & $* *$ \\
\hline & $\mathrm{TL}$ & $\mathrm{Y}=15.096+0.131 \mathrm{x}$ & 0.007 & $\mathrm{Ns}$ \\
\hline \multirow[t]{9}{*}{ West African Dwarf } & HL & $\mathrm{Y}=19.159+0.267 \mathrm{x}$ & 0.020 & Ns \\
\hline & EL & $Y=19.102-0.138 x$ & 0.005 & Ns \\
\hline & SW & $Y=14.148+0.216 x$ & 0.086 & Ns \\
\hline & $\mathrm{NC}$ & $Y=14.068+0.145 x$ & 0.023 & Ns \\
\hline & $\mathrm{BL}$ & $Y=14.263+0.069 x$ & 0.018 & Ns \\
\hline & WH & $Y=15.736+0.039 x$ & 0.011 & Ns \\
\hline & HG & $Y=16.818+0.015 x$ & 0.000 & Ns \\
\hline & PG & $Y=16.992+0.012 x$ & 0.000 & Ns \\
\hline & $\mathrm{TL}$ & $Y=17.157+0.043 x$ & 0.000 & $\mathrm{Ns}$ \\
\hline
\end{tabular}

**P $<0.01,{ }^{*} \mathrm{P}<0.05$, ns-not significant 
Table 5: Regression of liveweight on body parameters $(Y=$ live wieght $)$ in Does $>2$ y

\begin{tabular}{|c|c|c|c|c|}
\hline Breed & Parameter & Regression equation & $\mathbf{r}^{2}$ & Significance \\
\hline \multirow[t]{9}{*}{ Red Sokoto } & HL & $Y=17.329+0.217 x$ & 0.018 & $\mathrm{Ns}$ \\
\hline & EL & $Y=20.469+0.123 x$ & 0.007 & Ns \\
\hline & SW & $Y=16.569+0.121 x$ & 0.023 & Ns \\
\hline & $\mathrm{NC}$ & $Y=11.724+0.290 x$ & 0.099 & $*$ \\
\hline & $\mathrm{BL}$ & $Y=18.554+0.008 x$ & 0.000 & Ns \\
\hline & WH & $\mathrm{Y}=16.913+0.035 \mathrm{x}$ & 0.004 & Ns \\
\hline & $\mathrm{HG}$ & $Y=4.788+0.231 x$ & 0.106 & $*$ \\
\hline & PG & $Y=4.941+0.221 x$ & 0.096 & * \\
\hline & TL & $Y=17.364+0.120 x$ & 0.006 & Ns \\
\hline \multirow[t]{9}{*}{ Sahel } & HL & $Y=20.677+0.278 x$ & 0.024 & Ns \\
\hline & EL & $Y=30.436+-0.469 x$ & 0.658 & Ns \\
\hline & SW & $\mathrm{Y}=20.084+0.140 \mathrm{x}$ & 0.006 & Ns \\
\hline & $\mathrm{NC}$ & $Y=29.791+0.228 x$ & 0.040 & Ns \\
\hline & $\mathrm{BL}$ & $\mathrm{Y}=37.562+0.239 \mathrm{x}$ & 0.081 & * \\
\hline & WH & $\mathrm{Y}=3.424+0.383 \mathrm{x}$ & 0.141 & $* *$ \\
\hline & HG & $Y=-1.444+0.388 x$ & 0.074 & * \\
\hline & PG & $Y=-2.345+0.387 x$ & 0.075 & * \\
\hline & TL & $Y=25.151+-0.122 x$ & 0.002 & Ns \\
\hline \multirow[t]{9}{*}{ West African Dwarf } & HL & $Y=18.589+0.074 x$ & 0.006 & Ns \\
\hline & EL & $Y=18.737+0.035 x$ & 0.001 & Ns \\
\hline & SW & $\mathrm{Y}=19.817+-0.046 \mathrm{x}$ & 0.004 & Ns \\
\hline & $\mathrm{NC}$ & $Y=25.185^{+-0.219 x}$ & 0.076 & * \\
\hline & $\mathrm{BL}$ & $Y=16.788+0.053 x$ & 0.025 & Ns \\
\hline & WH & $\mathrm{Y}=18.907+0.005 \mathrm{x}$ & 0.000 & Ns \\
\hline & HG & $Y=13.215+0.086 x$ & 0.036 & Ns \\
\hline & PG & $Y=19.280+-0.002 x$ & 0.000 & Ns \\
\hline & TL & $Y=19.115+0.000 x$ & 0.000 & Ns \\
\hline
\end{tabular}

${ }^{* *} \mathrm{P}<0.01,{ }^{*} \mathrm{P}<0.05$, Ns-not significant

Table 6: Regression of liveweight on body parameters $(Y=$ live wieght $)$ in Bucks $>2$ y

\begin{tabular}{|c|c|c|c|c|}
\hline Breed & Parameter & Regression equation & $\mathbf{r}^{2}$ & Significance \\
\hline \multirow[t]{9}{*}{ Red Sokoto } & HL & $Y=15.698+0.482 x$ & 0.065 & $\mathrm{Ns}$ \\
\hline & EL & $Y=15.634+0.301 x$ & 0.018 & $\mathrm{Ns}$ \\
\hline & SW & $Y=18.283+0.046 x$ & 0.005 & Ns \\
\hline & $\mathrm{NC}$ & $Y=15.592+0.147 x$ & 0.014 & Ns \\
\hline & $\mathrm{BL}$ & $Y=21.053+0.040 x$ & 0.014 & Ns \\
\hline & WH & $Y=16.842+0.040 x$ & 0.005 & Ns \\
\hline & HG & $Y=27.291+0.133 x$ & 0.027 & Ns \\
\hline & PG & $Y=27.527+0.132 x$ & 0.026 & Ns \\
\hline & TL & $Y=17.974+0.099 x$ & 0.002 & Ns \\
\hline \multirow[t]{9}{*}{ Sahel } & HL & $Y=25.296+0.016 x$ & 0.000 & Ns \\
\hline & EL & $Y=30.547+-0.396 x$ & 0.025 & Ns \\
\hline & SW & $\mathrm{Y}=24.342+0.314 \mathrm{X}$ & 0.000 & Ns \\
\hline & $\mathrm{NC}$ & $Y=16.640+0.318 x$ & 0.057 & Ns \\
\hline & $\mathrm{BL}$ & $Y=29.716+0.082 x$ & 0.007 & Ns \\
\hline & WH & $Y=-18.636+0.608 x$ & 0.235 & $* *$ \\
\hline & HG & $Y=2.705+0.341 x$ & 0.024 & Ns \\
\hline & PG & $Y=23.921+0.018 x$ & 0.000 & Ns \\
\hline & $\mathrm{TL}$ & $Y=28.089+-0.230 x$ & 0.009 & Ns \\
\hline \multirow{9}{*}{ West African Dwarf } & HL & $Y=18.623+0.106 x$ & 0.015 & $\mathrm{Ns}$ \\
\hline & EL & $Y=17.310+0.191 x$ & 0.038 & $\mathrm{Ns}$ \\
\hline & SW & $Y=17.850+0.097 x$ & 0.028 & Ns \\
\hline & $\mathrm{NC}$ & $Y=21.303^{+-0.072 x}$ & 0.011 & Ns \\
\hline & $\mathrm{BL}$ & $Y=16.913+0.557 x$ & 0.024 & Ns \\
\hline & WH & $Y=20.337+-0.218 x$ & 0.005 & Ns \\
\hline & HG & $Y=15.703^{+-0.005 x}$ & 0.000 & Ns \\
\hline & PG & $Y=19.703+0.005 x$ & 0.000 & Ns \\
\hline & TL & $Y=23.016+0.307 x$ & 0.066 & $\mathrm{Ns}$ \\
\hline
\end{tabular}

${ }^{* *} \mathrm{P}<0.01,{ }^{*} \mathrm{P}<0.05$, ns-not significant 


\section{RESULTS AND DISCUSSION}

The regressions between bodyweight and body measurements of goats $<1 \mathrm{y}$ are shown in table 1 and 2 . Live weight changes with body measurements were poorly predictable in Red Sokoto does with $\mathrm{R}^{2}$ values ranging between 0.000-0.027. Also, all the body measurements were not significant $(\mathrm{p}>0.05)$ for both sexes. Five parameters HL, SW, NC, BL and WH for does and HL, SW, $\mathrm{NC}, \mathrm{BL}$ and $\mathrm{WH}$ for bucks were strongly predictable for live weight with $\mathrm{R}^{2}$ values ranging from 0.006-0.190 for does and 0.003-0.202 for bucks in Sahel breeds. In WAD breed, BL had a strong and predictive influence on live weight changes while other body measurements parameter was redundant for the does with $\mathrm{R}^{2}$ values ranging from 0.000-0.031. All body measurements in the buck showed poor prediction (0.003-0.035) and were generally redundant.

Table 3 and 4 shows the regression between bodyweight and body measurements between 1-2 y of age in different breeds. Live weight changes with body measurements were poorly predictable in Red Sokoto does with $\mathrm{R}^{2}$ values ranging between 0.000-0.241 and all the body measurements were not significant ( $\mathrm{p}>0.05)$. HL, BL and WH had a strong predictive power with a significant ( $p<0.05$ ) influence on bodyweight and $\mathrm{R}^{2}$ values ranging between 0.005-0.260 in the bucks. Five parameters such as HL, EL, HG, BL and WH for does and EL, HG, PG, BL and WH for bucks were strongly predictable for liveweight with $\mathrm{R}^{2}$ values ranging from 0.000-0.185 for does and 0.0000.164 for bucks in Sahel breeds. In WAD breed, four parameters such as HL, EL, BL and HG had a strong and significance influence on liveweight changes while other body measurements parameter were redundant for the does with $\mathrm{R}^{2}$ values ranging from 0.005-0.048. All body measurements in the buck showed low predictive power (o.000-0.086) and were generally not significant ( $\mathrm{p}>0.05)$.

The regressions between bodyweight and body measurements of goats studied above $2 \mathrm{y}$ of age are presented in table 5 and 6 . Live weight changes with body measurements were poorly predictable in Red Sokoto goatss with $\mathrm{R}^{2}$ values ranging between 0.002-0.065 for the does. Three variables such as NC, HG and PG had positive and significant $(\mathrm{p}<0.05)$ influence on liveweight with $\mathrm{R}^{2}$ values ranging between 0.000-0.106 for the bucks. Four parameters such as $\mathrm{HG}, \mathrm{PG}, \mathrm{BL}$ and $\mathrm{WH}$ for does and a single variable (BL) for bucks were strongly predictable for liveweight with $\mathrm{R}^{2}$ values ranging from 0.002-0.658 for does and 0.000-0.235 for bucks in Sahel breeds. In WAD breed, NC had a moderate predictive influence on liveweight changes while other body measurements parameter was redundant for the does with $\mathrm{R}^{2}$ values ranging from 0.000-0.076. All body measurements in the buck showed poor prediction (0.000-0.066) and were redundant.

The low, moderate and high predictive power obtained from this study might be due to instability of regression coefficient at different ages. The use of variables which are interdependent explanatory should be treated with caution as multicollinearity can be there with unstable estimates of regression coefficients [9], which in turn become impossible to estimate the unique effects of the predictors. Our results are in accordance with previous reports of Ojedapo et al. [7] and Leng et al. [5]. The importance of HG in weight estimation could be as a result of the fact that muscle, some fat along with bone structure contribute to its formation. However, Cam et al. [10] reported that the trait cannot be used to predict live weight accurately. Low predictive power from these findings lend credence that traits use as sole variable for prediction are not environmentally sensitive and therefore are indicators of inherent size. The better prediction observed with different breeds compared to their bucks in this study agreed with the findings of Ojedapo et al. [7] that sexual dimorphism in body weight and other body linear measurements favored females than males in goats. HG however, did not show sufficient predictions across the classes as expected of a major predictor [1-3].

\section{REFERENCES}

1. Thiruvenkadan, A. K. and Panneerselvam, S., (2005). Body weight and its association with body measurements in Kanni Adu goats. Veterinary Journal. (86), 487-90.

2. Salako, A. E. (2006). Application of morphological indices in the assessment of type and function in sheep. Int Journal of Animal Science. 111 (2013) 2330.

3. Mavule, B. S., Muchenje, V., Bezuidenhout, C. C. and Kunene, N. W. (2013). Morphological structure of Zulu sheep based on principal component analysis of body measurements. Small Ruminant Research 111 (2013) 2330

4. Baffour-Awuah, O., Ampofo, E., Dodoo, R. (2000). Predicting the live weight of sheep by using linear body measurements. Ghana J. Agric. Sci. 33, 207-212.

5. Leng J., Zhu, R., Zhao, G., Yang Q., Mao, H. (2010). Quantitative and qualitative body traits of Longling Yellow goats in China. Agricultural Sciences inChina,9:408-415.

6. Salako, A. E. and Ngere, L. O. (2002). Application of Multifactorial Discriminate analysis in the morphometric structural differentiation of West African Dwarf (WAD) and Yankassa Sheep in the Southwest. Nigerian Journal of Animal Production 29 (2): 163-167.

7. Ojedapo, L. O., Adedeji, T. A., Olayeni, T. B. Adedeji, O. S., Abdullah, A. R., Ojebiyi, O. O. (2007). Influence of age and sex on body weight and some body linear measurements of extensively reared wad goats in derived savannah zone of Nigeria. Journal of Animal Veterinar Advances. 6(1), 114-117.

8. SAS (1990). Statistical Analysis System (SAS). Institute Inc. (1990). SAS/STAT User's guide version $6,4^{\text {th }}$ edition Vol. 2 SAS Inst-Inc. carry, N. C 846.

9. Malau-Aduli, B. S., L. O. Eduvie, C. A. M. Lakpini and A. E. Malau-Aduli, (2003). Variations in live weight gains, milk yield and composition of Red Sokoto goats fed crop-residue-based supplements in the subhumid zone of Nigeria. Livestock Prod. Sci., 83:63-71.

10. Cam, M. A., M. Olfaz and E. Soydan. (2010b). Body measurements reflect body weights and carcass yields in Karayaka sheep. Asian J. Anim. Vet. Adv. 5(2):120-127. 